\title{
Aldose reductase functions as a detoxification system for lipid peroxidation products in vasculitis
}

\author{
Heike L. Rittner, ${ }^{1}$ Verena Hafner, ${ }^{1}$ Piotr A. Klimiuk, ${ }^{1}$ Luke I. Szweda, ${ }^{2}$ Jörg J. Goronzy, ${ }^{1}$ \\ and Cornelia M. Weyand ${ }^{1}$
}

\begin{abstract}
${ }^{1}$ Department of Medicine, Division of Rheumatology, Mayo Clinic, Rochester, Minnesota 55905, USA
${ }^{2}$ Department of Physiology and Biophysics, Case Western Reserve University, Cleveland, Ohio 44106-4970, USA

Address correspondence to: Cornelia Weyand, Mayo Clinic, 200 First Street SW, Guggenheim 401, Rochester, Minnesota 55905, USA. Phone: (507) 284-1650; Fax: (507) 284-5045; E-mail: weyand.cornelia@ mayo.edu
\end{abstract}

Received for publication September 24, 1998, and accepted in revised form February 11, 1999.

\begin{abstract}
Giant cell arteritis (GCA) is a systemic vasculitis preferentially affecting large and medium-sized arteries. Inflammatory infiltrates in the arterial wall induce luminal occlusion with subsequent ischemia and degradation of the elastic membranes, allowing aneurysm formation. To identify pathways relevant to the disease process, differential display-PCR was used. The enzyme aldose reductase (AR), which is implicated in the regulation of tissue osmolarity, was found to be upregulated in the arteritic lesions. Upregulated AR expression was limited to areas of tissue destruction in inflamed arteries, where it was detected in T cells, macrophages, and smooth muscle cells. The production of AR was highly correlated with the presence of 4-hydroxynonenal (HNE), a toxic aldehyde and downstream product of lipid peroxidation. In vitro exposure of mononuclear cells to HNE was sufficient to induce AR production. The in vivo relationship of AR and HNE was explored by treating human GCA temporal artery-severe combined immunodeficiency (SCID) mouse chimeras with the AR inhibitors Sorbinil and Zopolrestat. Inhibition of AR increased HNE adducts twofold and the number of apoptotic cells in the arterial wall threefold. These data demonstrate that AR has a tissue-protective function by preventing damage from lipid peroxidation. We propose that $\mathrm{AR}$ is an oxidative defense mechanism able to neutralize the toxic effects of lipid peroxidation and has a role in limiting the arterial wall injury mediated by reactive oxygen species.
\end{abstract}

J. Clin. Invest. 103:1007-1013 (1999).

\section{Introduction}

Giant cell (temporal) arteritis (GCA) is an inflammatory vasculopathy that manifests as a systemic disease (1, 2 ). The primary sites of inflammation are the walls of large and medium-sized arteries. T cells, macrophages, and multinucleated giant cells infiltrate into all layers of the arterial wall and form tissue-destructive granulomas in the media and at the media-intima junction. Accumulating data suggest that GCA is a $\mathrm{T}$ celldependent disease (3), and evidence has been provided that the disease-inducing antigen resides in the arterial wall (4). Antigen recognition appears to occur distant from the focus of arterial wall damage, namely, in the adventitia of affected arteries (5).

Mechanisms of tissue destruction in GCA are beginning to be understood. Luminal occlusion caused by the formation of neointima causes blindness and stroke. Intimal hyperplasia in inflamed arteries is closely correlated with the production of platelet-derived growth factor (6), which is preferentially supplied by multinucleated giant cells and macrophages sitting next to the internal elastic lamina. Different mechanisms are involved in the destruction of the arterial muscle layer and the fragmentation of elastic fibers. Necrosis of medial smooth muscle cells (SMCs) and degradation of the elastic laminae dominate the picture in large-vessel arteritis, particularly in GCA aortitis. Production of matrix metalloproteinases (MMPs), such as MMP-2 (7) and MMP-9 (8), has been implicated in the fragmentation of the internal elastic membrane. Production of nitric oxide synthase- 2 by intimal macrophages may also have a role in tissue injury (7). In addition, we have recently suggested (9) that free oxygen radicals produced by macrophages contribute to the medial damage in affected arteries. Free radicals have a very short half-life, and direct detection of them in tissue is very difficult. However, free radicals initiate lipid peroxidation, which results in the formation of stable aldehydes such as 4hydroxynonenal (HNE) and malon dialdehyde (MDA) (10). These toxic aliphatic aldehydes are highly active and diffusible and are considered secondary cytotoxic messengers. HNE interacts destructively with proteins and nucleotides to form adducts, which can be identified in tissue by specific antibodies (11). Such lipid peroxidation products detected with HNE-reactive antibodies were abundantly present in tissue sections from GCA arteries. Notably, areas of pronounced tissue derangement in the media were characterized by strong HNE expression, indicating that oxygen radical-mediated tissue damage represents an important component of GCA.

Here, we report that vasculitic lesions, particularly in areas of prominent tissue injury, are also characterized by the upregulation of aldose reductase (AR). AR is a member of the aldo-keto reductase superfamily (12). It is a monomeric NADPH-dependent oxidoreductase with broad substrate specificity for carbonyl compounds (13). 
The enzyme has been implicated in causing secondary complications of diabetes mellitus (14). So far, a role of AR in immune responses or inflammation has not been established. Upregulation of AR in GCA was limited to areas of high HNE production, raising the possibility of a functional relationship. In in vitro experiments, HNE induced the production of AR in mononuclear cells. To explore the relationship of AR and HNE in arterial inflammation, human temporal artery-severe combined immunodeficiency (SCID) mouse chimeras were treated with the AR-specific inhibitors Sorbinil and Zopolrestat (15). AR inhibition resulted in a significant increase in HNE in the arterial wall combined with a threefold rise in the frequency of apoptotic cells in the tissue. We propose that AR functions as a detoxification system that degrades toxic aldehydes formed during oxidative damage in GCA.

\section{Methods}

Temporal artery samples. Temporal artery samples were obtained from routine diagnostic biopsies. The diagnosis of GCA was made in 22 patients with typical inflammatory infiltrates. Tissue specimens from control patients had no infiltrates in multiple serial sections. As well, control patients lacked clinical evidence of polymyalgia rheumatica. Specimens were snap-frozen in liquid nitrogen until used for RNA extraction or embedded in Tissue-Tek OCT compound (Sakura Finetek, Torrance, California, USA) for immunohistochemistry analysis.

Differential display-PCR. Gene expression was analyzed by differential display-PCR in temporal artery specimens from five patients with GCA and five control patients without vasculitis as described previously (9). Briefly, cDNA was amplified under nonstringent PCR conditions $\left(94^{\circ} \mathrm{C}\right.$ for $2 \mathrm{~min}, 40^{\circ} \mathrm{C}$ for $2 \mathrm{~min}, 72^{\circ} \mathrm{C}$ for $30 \mathrm{~s}$; 40 cycles) using a combination of ten $5^{\prime}$-random primers and four 3 '-oligo dT primers in the presence of $\left[\alpha-{ }^{33} \mathrm{P}\right]-\mathrm{dATP}(\mathrm{Du}$ Pont NEN Research Products, Boston, Massachusetts, USA). Bands differentially expressed between the temporal arteries from patients with GCA and control specimens were excised, eluted, and reamplified. Products were cloned into the PCR 2.1 vector (Invitrogen Corp., Carlsbad, California, USA), and the inserted PCR products were analyzed by automated sequencing.

$P C R$. Total RNA was extracted from temporal arteries using a commercially available kit (Trizol; Life Technologies Inc., Grand Island, New York, USA). cDNA from four different GCA arteries and four negative arteries were amplified under non-

Table 1

$A R$ is upregulated in areas of HNE formation

\begin{tabular}{|c|c|c|c|c|c|c|}
\hline & \multicolumn{2}{|c|}{ Intima } & \multicolumn{2}{|c|}{ Media } & \multicolumn{2}{|c|}{ Adventitia } \\
\hline & $\mathrm{AR}$ & HNE & AR & HNE & AR & $\mathrm{HN}$ \\
\hline \multicolumn{7}{|c|}{ Patient } \\
\hline 1 & 3.6 & 6.9 & 17.7 & 23.8 & 3.6 & 3.3 \\
\hline 2 & 2.1 & 3.9 & 30.0 & 36.2 & 3.8 & 3.8 \\
\hline 3 & 29.6 & 22.5 & 53.9 & 29.2 & 12.5 & 7.6 \\
\hline 4 & 7.3 & 3.7 & 38.8 & 25.2 & 13.9 & 9.7 \\
\hline 5 & 4.5 & 3.3 & 29.9 & 24.7 & 8.7 & 3.5 \\
\hline 6 & 10.8 & 8.3 & 32.8 & 29.8 & 4.7 & 8.7 \\
\hline Mean & 9.6 & 8.1 & 33.9 & 28.1 & 7.9 & 6.1 \\
\hline
\end{tabular}

Arterial sections from patients with GCA were stained with anti-AR and anti-HNE antibodies, respectively. Values are the percentages of the areas stained per entire arterial layer. $A R$, aldose reductase; GCA, giant cell arteritis; HNE, 4-hydroxy nonenal.
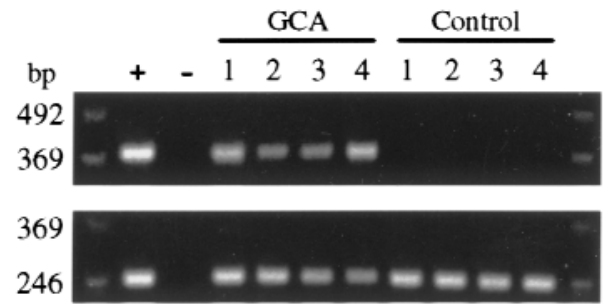

\section{Figure 1}

AR mRNA is upregulated in inflamed temporal arteries. cDNA from temporal arteries of four patients with giant cell arteritis (GCA) and four temporal arteries without histological signs of inflammation were amplified with AR-specific (top) and $\beta$-actin-specific primers (bottom). AR-specific sequences could not be detected in normal arteries but were abundantly expressed in inflamed arteries. $A R$, aldose reductase.

saturating conditions with $\beta$-actin (ATG GCC ACG GCT GCT TCC AGC; CAT GGT GGT GCC GCC AGA CAG)- and AR (GTG CAC GTA CCA TGA GAA GG; CTT TGG ACT GGC AGT ACT GG)-specific primers by PCR as described (16).

Immunohistochemistry. Single- and dual-color immunohistochemistry was performed on frozen sections of temporal arteries as described (5). Mouse monoclonal antibodies against CD3 (1:100) or CD68 (KP1) (1:250) and biotinylated anti-mouse Ig and anti-rabbit Ig antibodies were purchased from DAKO Corp. (Carpinteria, California, USA). A mouse monoclonal antibody against myosin heavy chain in smooth muscles was obtained from Sigma Chemical Co. (St. Louis, Missouri, USA). A polyclonal rabbit antibody against AR (1:500) was generously provided by R. Sorenson (University of Minnesota, Minneapolis, Minnesota, USA) (17).

To semiquantify the extent of lipid peroxidation in the arterial wall, tissue sections stained with anti-HNE antibodies and developed with the immunofluorescent Vector red (Vector Laboratories, Burlingame, California, USA) were scanned with a confocal laser microscope, and stained areas were determined with the Kontron Imaging System KS400 (Kontron Elektronik, Eiching, Germany). For topographic localization of stained cells, the percentage of stained area was calculated in each of the three arterial wall layers (intima, media, and adventitia).

Apoptosis assays. Apoptosis in frozen tissue sections was detected by Klenow end-labeling of blunt ends of doublestranded DNA fragments with biotinylated nucleotides (TACS I Klenow Kit; Trevigen, Gaithersburg, Maryland, USA). Apoptotic cells were developed with streptavidin-conjugated horseradish peroxidase and diaminobenzidine as substrate. The number of apoptotic cells was counted in five serial circumferential sections of the arteries.

Induction of $A R$. Protected HNE was converted into the unprotected form, and concentrations were calculated. Peripheral blood mononuclear cells (PBMCs) from healthy donors were isolated using Ficoll-Paque (Pharmacia Biotech, Piscataway, New Jersey, USA), washed twice in serum-free RPMI-1640, and $10^{6}$ cells were stimulated with 5 or $10 \mu \mathrm{M}$ $\mathrm{HNE}$ and $600 \mathrm{U} / \mathrm{ml}$ recombinant human interferon- $\gamma(\mathrm{IFN}-\gamma)$ (Genzyme Diagnostics, Cambridge, Massachusetts, USA) in RPMI-1640 for 6 h. Control samples were incubated with 200 $\mu \mathrm{M}$ Sorbinil. Cells were lysed and $2 \mu \mathrm{g}$ lysate was separated on $10 \%$ SDS-polyacrylamide gels. Proteins were transferred onto Immobilon-P PVDF membranes (Millipore Corp., Bedford, Massachusetts, USA) for $1 \mathrm{~h}(100 \mathrm{~V})$ at $4^{\circ} \mathrm{C}$. Membranes were blocked with $2 \%$ BSA and incubated overnight at $4{ }^{\circ} \mathrm{C}$ with a rabbit polyclonal anti-AR antibody $(1: 1,000)$. Proteins were detected using a horseradish peroxidase-conjugated goat anti-rabbit polyclonal antibody (Santa Cruz Biotechnology 

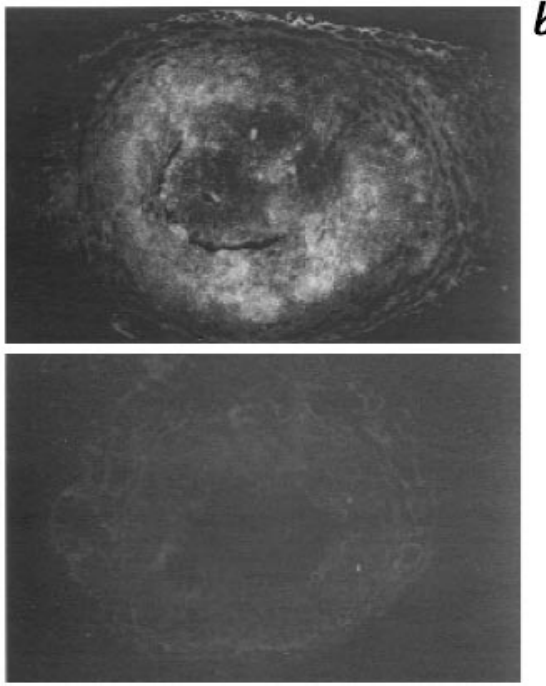

Figure 2

Mononuclear cells and smooth muscle cells in the media express AR. (a) Frozen sections from temporal arteries of patients with GCA and control patients were immunostained with a rabbit polyclonal AR-specific antibody (fluorescent Vector red). Widespread expression of AR in the medial layer was seen in GCA arteries (top). In contrast, AR was virtually absent in unaffected arteries (bottom). Original magnification: $\times 50$. (b) To determine the cell type expressing AR, temporal artery sections were double-stained with a rabbit polyclonal AR-specific antibody (red) and mouse monoclonal antibody against CD3 or CD68 (brown). A fraction of all cell types in the media, including $C D 3^{+} \mathrm{T}$ cells $($ top $)$ and $\mathrm{CD}^{+} 8^{+}$macrophages (bottom), expressed AR in areas of prominent tissue destruction. Original magnification: $\times 1,000$.
Inc., Santa Cruz, California, USA) and the ECL System (Amersham Life Sciences Inc., Arlington Heights, Illinois, USA).

Treatment of temporal artery-SCID mouse chimeras. Segments of temporal arteries from patients with GCA or control patients were subcutaneously implanted into NOD-SCID mice (The Jackson Laboratory, Bar Harbor, Maine, USA) as described previously (16, 18). Starting on day 8 after implantation, engrafted mice were injected intraperitoneally for 1 week with $50 \mathrm{mg} / \mathrm{kg} /$ day of Sorbinil or $50 \mathrm{mg} / \mathrm{kg} /$ day of Zopolrestat (both from Pfizer Inc., Groton, Connecticut, USA) in sterile $10 \%$ DMSO/PBS. The control group was sham-injected with solvent only. After treatment, the temporal arteries were explanted and embedded in OCT compound for immunohistochemical analysis.

HNE measurement. HNE was measured using a lipid peroxidation kit (Calbiochem-Novabiochem Corp., San Diego, California, USA). Kidneys from Sorbinil-treated and untreated SCID mice were homogenized in ice-cold $20 \mathrm{mM}$ Tris- $\mathrm{HCl}$ buffer ( $\mathrm{pH}$ 7.4). Samples were adjusted to $0.2 \mathrm{mg}$ protein (BioRad Laboratories Inc., Hercules, California, USA). After addition of a chromophore, the samples were incubated at $45^{\circ} \mathrm{C}$ for $40 \mathrm{~min}$. An HNE standard was run in parallel. After centrifugation, the absorbance of the supernatants was measured at $586 \mathrm{~nm}$, and the protein concentration was calculated by comparison with the HNE standard.

Statistical analysis. All statistical analyses were performed using SigmaStat software (SPSS Inc., Chicago, Illinois, USA). Samples were compared using the paired Student's $t$ test.

\section{Results}

$A R$ is upregulated in GCA. Differential display-PCR was used to identify gene products involved in arterial wall inflammation. Control tissues were analyzed in comparison with affected temporal arteries and with polyclonally stimulated PBMCs to bias the set of identified genes against activation-induced products of lymphocytes and macrophages. Twenty-two sequences were isolated using this approach; 11 could be matched with genes described previously (9). Sequence analysis of a differentially expressed 517-bp PCR product revealed a 98.8\% homology with the AR (E.C.1.1.1.2) gene at the 3' end of the cDNA sequence. Two additional differentially expressed genes, NADH dehydrogenase subunit 4 and cytochrome oxidase subunit 1 , were also related to cell biology of oxidative stress. To confirm the differential expression of AR in vascular lesions, cDNA was prepared from a set of four temporal artery specimens affected by GCA and four control temporal arteries and analyzed by PCR with AR-specific primers (Figure 1). All samples were adjusted for $\beta$-actin content. Transcripts for the AR gene were not detectable in lysates from noninflamed arteries but were strongly positive in temporal artery tissue from patients with GCA (Figure 1).

$A R$ is upregulated in macrophages, Tcells, and SMCs in areas of arterial wall destruction. AR is primarily expressed in the kidney (19) but has also been detected in the eye, the central and peripheral nervous systems, and the reproductive system. Endothelial cells and SMCs in blood vessels can produce the enzyme (20). To identify the cellular source of AR in GCA, tissue sections from inflamed and control temporal arteries were examined by immunofluorescence using an AR-specific antibody (Figure 2a). In control arteries, staining for AR was virtually absent. In contrast, the enzyme was strongly expressed in inflamed arteries. AR was not upregulated in all arterial wall layers. Very few cells in the adventitia and the intima of inflamed arteries expressed AR. High expression of AR was found in the media, with a patchy distribution of positive and negative regions. Areas with upregulation of AR were characterized by extensive tissue damage, as judged by the destruction of medial SMCs and the fragmentation of the elastic laminae bordering the media. Cell types contributing to the expression of AR were identified by two-color immunohistochemistry. In regions of expression, $\mathrm{AR}$ was found in $\mathrm{CD}^{+} \mathrm{T}$ cells, $\mathrm{CD}^{+} 8^{+}$macrophages, and SMCs (Figure $2 b$ ). AR was consistently detected in multinucleated giant cells, which are the histological hallmark of GCA and are a component of the intramural granulomas formed in this arteritis.

The spatial correlation of AR expression and arterial wall damage suggested a possible relationship between tissue injury and upregulation of the enzyme. We have recently demonstrated (9) that HNE adducts are formed in the arteritic lesions, indicating a role of oxygen radicals in tissue destruction. To examine the relationship between AR expression and HNE formation in the arterial wall, adjacent tissue sections from the temporal arteries of six 

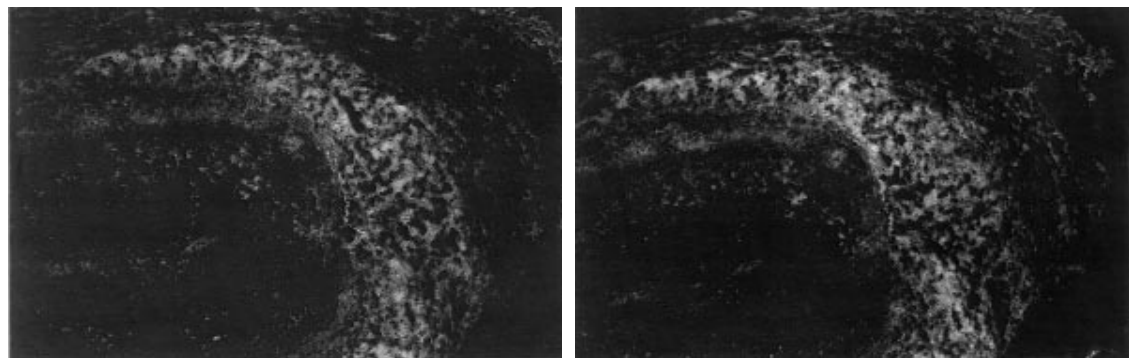

\section{Figure 3}

AR colocalizes with HNE. Adjacent frozen sections from six patients with GCA were labeled with either a rabbit polyclonal antibody against HNE adducts (as a marker for lipid peroxidation) or with an anti-AR antibody. Expression of AR (left) in the media closely correlated with signs of lipid peroxidation and HNE formation (HNE adducts) (right). Original magnification: $\times 100$. HNE, 4-hydroxynonenal.

patients were stained with antibodies specific for AR and HNE adducts (Figure 3 ). The stained areas in the adventitia, media, and intima were quantified by confocal laser microscopy, and the percentage of stained area to total area was calculated (Table 1). The presence of HNE correlated highly with the upregulation of $\operatorname{AR}\left(r^{2}=0.88, P<0.0001\right)$.

$H N E$ can induce AR in lymphocytes. The close correlation between AR and HNE in the arterial wall raised the question of whether HNE can affect the expression of AR. To approach this question, PBMCs were incubated with $\mathrm{HNE}$, and the expression of AR protein was monitored. PBMCs were incubated with 5 and $10 \mu \mathrm{M}$ HNE. HNE rapidly induced the synthesis of AR (Figure 4). A 36-kDa band staining with an AR-specific antibody could be detected in PBMCs six hours after their exposure to HNE. AR upregulation after stimulation of PBMCs with optimal amounts of IFN- $\gamma$ was detectable but less significant than after HNE. These data demonstrated that the lipid peroxidation product HNE induced the synthesis of AR in mononuclear cells, suggesting that the emergence of AR in vascular lesions of GCA was a consequence of lipid peroxidation.

Inbibition of AR in temporal artery-SCID mouse chimeras augments HNE formation and cell death in the arterial grafts. The induction of AR by the toxic aldehyde HNE raised the question of how this enzyme affected lipid peroxidation and the resulting tissue injury in vivo. We have recently established an in vivo model for GCA by implanting inflamed human temporal arteries into SCID mice. The disease is maintained in the arterial grafts, and mechanisms of therapeutic interventions can be explored by treating the temporal artery-SCID mouse chimeras (16, 18). To investigate directly the role of AR upregulation in

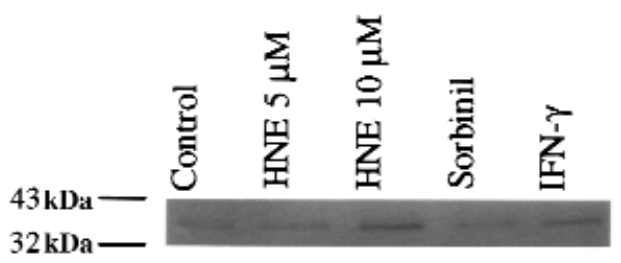

\footnotetext{
Figure 4

4-hydroxynonenal (HNE) induces AR in mononuclear cells. PBMCs were stimulated with 5 or $10 \mu \mathrm{M} \mathrm{HNE}$ in serum-free RPMI- 1640 for $6 \mathrm{~h}$. Protein $(2 \mu \mathrm{g})$ was separated on $10 \%$ SDS-polyacrylamide gels, and AR was detected by Western blotting. Control samples included a buffer control, $200 \mu \mathrm{M}$ Sorbinil, and $600 \mathrm{U} / \mathrm{ml}$ recombinant human interferon- $\gamma$ (IFN$\gamma)$, respectively. One experiment representative of eight is shown. PBMC, peripheral blood mononuclear cell.
}

the arterial wall, we took advantage of the AR-specific inhibitors, Sorbinil and Zopolrestat. Both compounds are well-characterized, noncompetitive inhibitors of AR that have been used in animal models and clinical trials $(15,21)$. Temporal arteries from patients with GCA were engrafted subcutaneously into SCID mice. The treatment group was injected intraperitoneally with $50 \mathrm{mg} / \mathrm{kg} /$ day Sorbinil or Zopolrestat for one week, and the control group received sham injections. The temporal arteries were then retrieved. Staining with antibodies to AR showed that the expression of AR was maintained in the arteries harvested from treated mice (Figure 5, $a$ and $b$ ). In addition to the tissue grafts, the kidneys of the control and treated animals were also harvested.

To determine the effect of AR on HNE formation in vivo, HNE concentrations in the renal tissue were measured. HNE was detectable in the kidneys from the controls but, upon inhibition of AR by Sorbinil, the mean HNE concentration increased significantly from $4.0 \mu \mathrm{M}$ to $6.4 \mu \mathrm{M}$ $(P=0.001)$ (Figure 5c). This finding indicated that expression of AR and the generation of the toxic aldehyde HNE were interrelated and that AR inhibition in vivo increased downstream products of lipid peroxidation.

To explore the in vivo function of AR in arteritis, sections of temporal artery grafts collected from Sorbinil-, Zopolrestat-, and sham-treated animals were stained for HNE adducts. Tissue areas stained with an HNE-specific antibody were quantified by confocal laser microscopy. As shown in Figure $5 d$, inhibition of AR in the Sorbinil-treated mice nearly doubled the HNE content in the arterial wall in comparison with the control mice $(7.1 \pm 1.8 \%$ vs. 3.9 $\pm 1.3 \%$, respectively; $P=0.028$ ). Treatment with Zopolrestat resulted in a threefold increase in HNE-stained areas (Figure $5 e ; 13.1 \pm 4.6 \%$ vs. $4.4 \pm 4.6 \% ; P=0.002$ ).

HNE can function as a cytopathic substance for almost all cell types (22). Given that HNE production was enhanced after the inhibition of AR, the temporal artery tissue was evaluated for evidence of cytotoxicity. The number of apoptotic cells was quantified in tissue sections of artery grafts from four untreated and four Sorbinil-treated temporal artery-SCID mouse chimeras using the in situ end-labeling (ISEL) method (Figure 6). In tissue sections from control grafts, a mean of $42 \pm 19$ apoptotic cells per arterial circumference was found. This number increased by more than threefold to an average of $143 \pm 38$ apoptotic cells per section of artery in the Sorbinil-treated grafts $(P=0.003)$. Apoptotic cells included SMCs as well as mononuclear cells (Figure $6 a$, left). Similar results were obtained with the second AR inhibitor, Zopolrestat (Figure 6b; $142 \pm 33$ vs. $36 \pm 16$ 

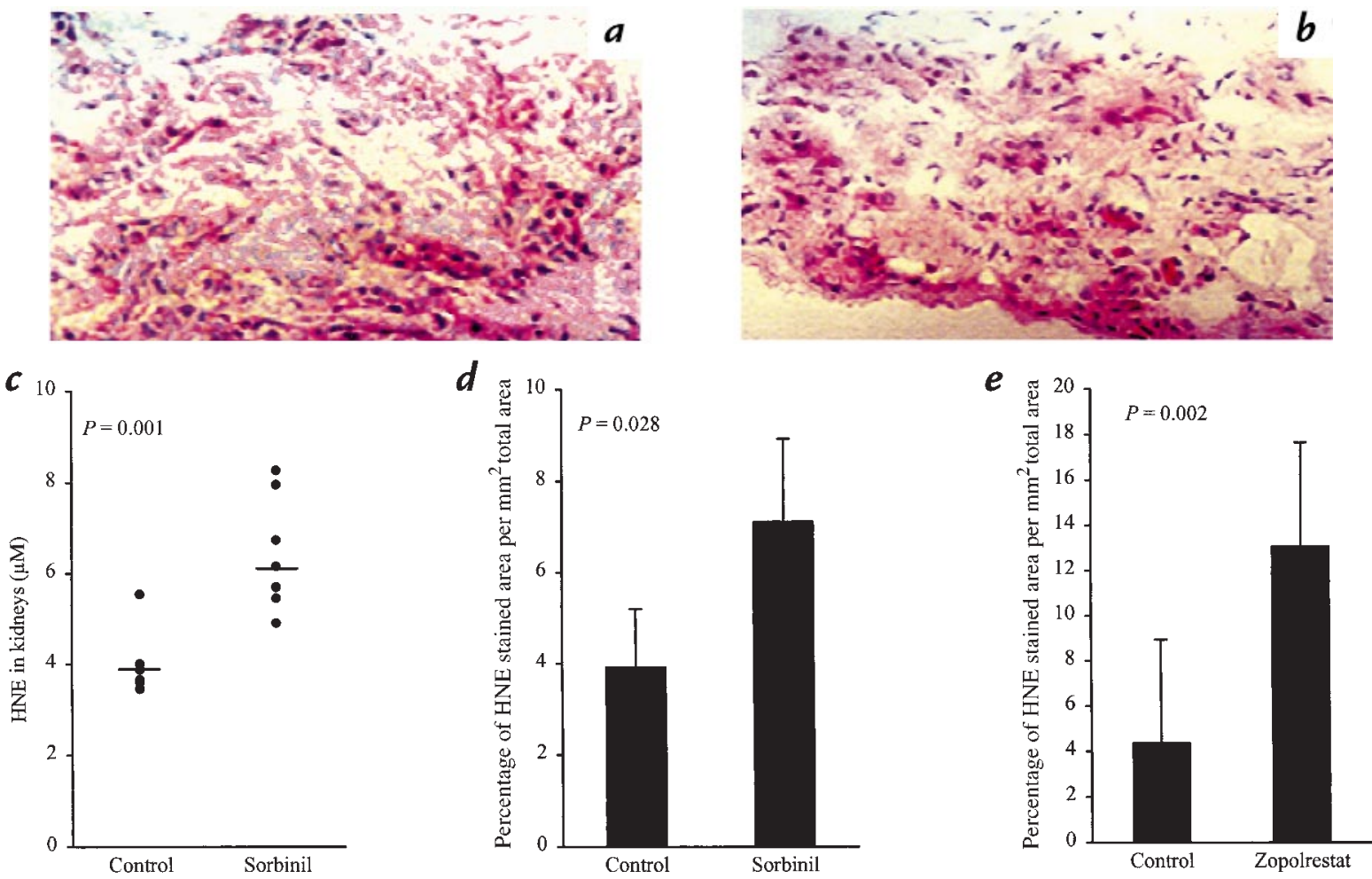

Figure 5

Treatment of temporal artery-SCID mouse chimeras with AR inhibitors increases the lipid peroxidation downstream product HNE. Temporal artery samples from patients were transplanted subcutaneously into SCID mice. Temporal artery-SCID mouse chimeras were injected with either Sorbinil $(50 \mathrm{mg} / \mathrm{kg} /$ day) or Zopolrestat $(50 \mathrm{mg} / \mathrm{kg} /$ day) intraperitoneally for 1 week or were sham-treated. The temporal artery grafts were explanted after treatment and stained with antibodies to AR as described in Figure 2. AR expression (red stain) was detected in sections from sham-treated (a) as well as from Sorbinil-treated $(\boldsymbol{b})$ tissues. Original magnification: $\times 400$. (c) Kidneys were harvested after completion of the treatment, and tissue extracts were adjusted to $0.2 \mathrm{mg}$ protein. HNE in the renal tissue was measured by a lipid peroxidation assay. ( $\boldsymbol{d}$ and $\boldsymbol{e}$ ) Frozen sections were stained by immunofluorescence with an HNE adduct-specific antibody. The stained area was quantified by laser confocal microscopy, and the percentage of stained tissue in relation to the entire arterial circumference was calculated. SCID, severe combined immunodeficiency.

apoptotic cells). Temporal arteries from sham treated GCA-SCID chimeras showed only little apoptosis (Figure $6 a$, center). To rule out the possibility that the increased apoptosis in situ was a direct consequence of Sorbinil treatment, the cytotoxic potential of Sorbinil itself was examined. PBMCs were incubated with Sorbinil at a concentration of $200 \mu \mathrm{M}$, and the fraction of subdiploid cells was determined by 7-amino-actinomycin D staining. A possible antiproliferative effect of Sorbinil on polyclonally activated PBMCs was explored by $\left[{ }^{3} \mathrm{H}\right]$ thymidine incorporation. No effect of Sorbinil on either cell proliferation or the number of apoptotic cells was found (data not shown). Temporal artery specimens from control patients were implanted into SCID mice and the mice treated with Sorbinil. Induction of apoptosis in SMCs was not observed (Figure 6a, right).

\section{Discussion}

The data presented here establish that the enzyme AR exhibits cytoprotective functions in GCA, an inflammatory blood vessel disease in which oxidative damage is an important pathway of tissue injury. The most susceptible targets of oxidative damage are polyunsaturated fatty acids, and major downstream products of lipid peroxidation are HNE adducts (10). In GCA, HNE adducts are detected in areas of prominent tissue damage, with medial SMCs, T cells, and macrophages as the most-affected cell populations. Here, we demonstrate that the toxic aldehyde HNE not only promotes cell death but also induces the expression of AR. From the experiments described, it can be concluded that AR reduces HNE in vivo and thus protects the tissue from cytopathic effects. The ability of AR to limit the in vivo availability of HNE and to provide protection from cytotoxic effects suggests that AR is an oxidative defense system that can be therapeutically explored.

$\mathrm{AR}$ is a member of the aldo-keto reductase superfamily (12), which catalyzes the reduction of a broad range of aldehydes (23). Its pattern of tissue expression with preferential production in the renal medulla has suggested a contribution in the regulation of tissue osmolarity (24), and an osmotic response element has been identified in the promoter region of $\operatorname{AR}(25,26)$. The enzyme has also been detected in the Schwann cell sheath of peripheral nerves, the lens, the retina, and in arterial endothelium $(14,17,19,20,27,28)$. Although the physiological role of AR in these tissues is not completely understood, the enzyme has attracted attention as a potential culprit in complications of diabetes, particularly diabetic neuropathy (29). AR catalyzes the reduction of glucose to sorbitol, an osmotically active substance associated with diabetic complications in the retina, lens, and peripher- 

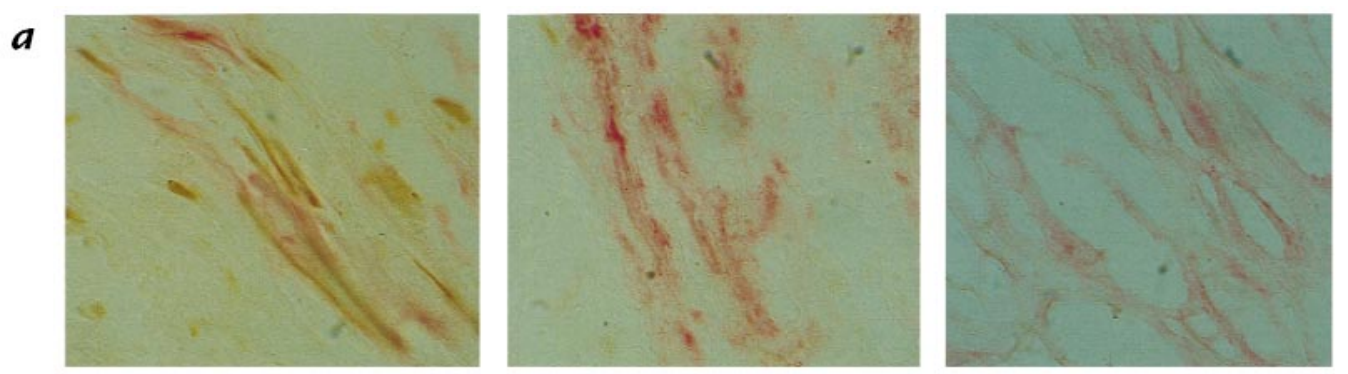

$\boldsymbol{b}$
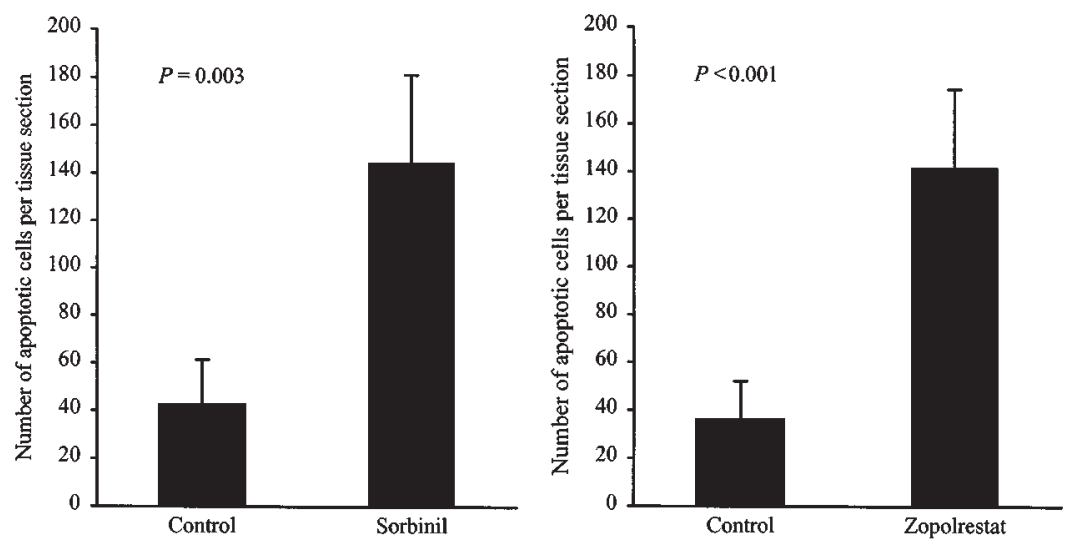

Figure 6

Treatment of temporal artery-SCID mouse chimeras with AR inhibitors leads to increased apoptosis. Temporal artery tissue from patients with GCA was implanted into SCID mice, and the chimeras were treated with Sorbinil, Zopolrestat, or buffer control as described in Figure 5. Apoptosis in the arterial wall was detected with ISEL staining in tissue sections from grafts explanted from control and Sorbinil- or Zopolrestat-treated SCID mouse chimeras. (a) Apoptotic smooth muscle cells were found in arterial sections of grafts harvested from Sorbinil-treated mice (left) but not in inflamed temporal arteries collected from sham-treated mice (middle) or in tissue sections of noninflamed control arteries explanted from Sorbinil-treated mice (right). ( $\boldsymbol{b}$ ) The total number of apoptotic cells in the entire arterial circumference was determined. Treatment with Sorbinil (left) as well as Zopolrestat (right) increased the number of apoptotic smooth muscle cells and apoptotic mononuclear cells in comparison to sham-treated controls. ISEL, in situ end labeling.

al nervous system. Consequently, Sorbinil was developed for clinical trials with the goal of preventing complications from hyperglycemia. However, no clear-cut therapeutic benefits have been demonstrated in these experimental therapies (30), raising questions concerning the exact role of AR in diabetes-related tissue injury. Data presented here emphasize that AR can function as a beneficial and not a tissue-injurious system. Together with the lack of therapeutic benefit from AR inhibition, it might be warranted to reconsider the role of AR in diabetic tissue damage.

So far, AR has not been associated with the immune system or inflammatory reactions. The isolation of the AR gene from inflamed temporal arteries was the first clue that $\mathrm{AR}$ might have a role in inflammation. Immunohistochemical analysis confirmed the upregulation of AR in the vascular lesions and demonstrated that the enzyme had a distinct tissue distribution. The close spatial relationship between AR upregulation and media damage provided the incentive to assess a possible role of the enzyme in arterial wall injury. The high expression of $\mathrm{AR}$ in regions of tissue necrosis and derangement could have suggested involvement of AR in injurious reactions, quite parallel to the suspected role of $\mathrm{AR}$ in diabetes. However, the tissue expression of AR mimicked the distribution of HNE, raising the possibility of a relationship between these two systems. HNE, being an aldehyde, is a possible substrate for AR, and AR can catalyze HNE degradation (31-33). We also considered the possibility that HNE could induce AR synthesis. Spycher et al. (34) have reported that AR production is induced in SMCs exposed to oxidative stress by hydrogen peroxide and have identified HNE as one important regulator of AR synthesis. In an extension of these studies, treatment of PBMCs with HNE led to the prompt induction of AR (Figure 4). IFN- $\gamma$, as the major tissue cytokine in GCA, is less effective in inducing AR expression. Moreover, IFN- $\gamma$ is only produced in the adventitia of GCA vessels and not in the media, where AR expression and lipid peroxidation is found. The unusual expression of AR in tissue-infiltrating $\mathrm{T}$ cells, $\mathrm{CD}^{+} 8^{+}$macrophages, and SMCs in the arterial media can thus be interpreted as a consequence of local HNE formation.

The increased HNE production upon AR inhibition in vivo is consistent with a major role of the enzyme in HNE degradation. This action was not limited to inflammatory lesions but could also be demonstrated in renal tissue. In the inflammatory lesions, AR inhibition led to amplified tissue destruction and established that AR can function as an oxidative defense enzyme. After one week of Sorbinil-mediated or Zopolrestat-mediated AR inhibition, the arterial architecture in implanted temporal artery grafts was seriously damaged and HNE staining in the tissue increased markedly. Increased tissue concentrations of HNE were correlated with a threefold increase in the number of apoptotic cells in the arterial 
wall. Increased susceptibility to apoptosis involved tissue-infiltrating mononuclear cells as well as SMCs (Figure $6 a$ ). This observation is consistent with the hypothesis that $\mathrm{AR}$ is an important self-protection system for $\mathrm{T}$ cells and macrophages in inflammatory processes. Macrophages appear to be a major source of oxygen radicals, initiating lipid peroxidation, cell membrane injury, and death (35). It is therefore possible that macrophages protect themselves from the cytopathic effects of their products by upregulating AR.

Several enzymes, including glutathione transferase, aldehyde dehydrogenase, and alcohol dehydrogenase, have been implicated in the detoxification of HNE. Most of these enzymes are present at highest concentration in the liver (36). Other metabolic pathways might have a role in detoxification reactions for saturated aldehydes produced by lipid peroxidation (37). The unique finding of our study is that AR can directly function as an HNEdetoxifying enzyme in inflammatory lesions. Such a mechanism not only provides beneficial effects for tissues attacked by oxygen radicals but also prevents suicide of inflammatory cells initiating oxidative stress.

The action of AR as a detoxifying enzyme for toxic aldehydes is unlikely to be a phenomenon limited to the inflammation in GCA. Rather, it can be predicted that AR expression is also upregulated in other inflammatory diseases associated with oxidative stress and local production of HNE. It is therefore possible that this mechanism of tissue protection is used in other inflammatory conditions and could be used therapeutically.

\section{Acknowledgments}

We thank our colleagues from the Department of Ophthalmology for their support, and the patients who donated the tissue to make these studies possible. We appreciate the help of James Fulbright and Toni L. Higgins in preparing the manuscript. This work was supported in part by a grant from the National Institutes of Health (R01 EY 11916) and by fellowships from the Deutsche Forschungsgemeinschaft (RI-817/1; to H.L. Rittner) and the Fogarty International Center, National Institutes of Health (F05 TWO5204; to P.A. Klimiuk).

1. Weyand, C.M., and Bartley, G.B. 1997. Giant cell arteritis: new concepts in pathogenesis and implications for management. Am. J. Ophthalmol. 123:392-395.

2. Hunder, G.G. 1997. Giant cell arteritis and polymyalgia rheumatica. Med. Clin. North Am. 81:195-219.

3. Weyand, C.M., and Goronzy, J.J. 1995. Giant cell arteritis as an antigendriven disease. Rheum. Dis. Clin. North Am. 21:1027-1039.

4. Martinez-Taboada, V., Hunder, N.N., Hunder, G.G., Weyand, C.M., and Goronzy, J.J. 1996. Recognition of tissue residing antigen by T cells in vasculitic lesions of giant cell arteritis. J. Mol. Med. 74:695-703.

5. Wagner, A.D., Bjornsson, J., Bartley, G.B., Goronzy, J.J., and Weyand, C.M. 1996. Interferon-gamma-producing $T$ cells in giant cell vasculitis represent a minority of tissue-infiltrating cells and are located distant from the site of pathology. Am. J. Pathol. 148:1925-1933.

6. Kaiser, M., Weyand, C.M., Bjornsson, J., and Goronzy, J.J. 1998. Platelet derived growth factor, intimal hyperplasia, and ischemic complications in giant cell arteritis. Arthritis Rheum. 41:623-633.

7. Weyand, C.M., Wagner, A.D., Bjornsson, J., and Goronzy, J.J. 1996. Correlation of the topographical arrangement and the functional pattern of tissue-infiltrating macrophages in giant cell arteritis. J. Clin. Invest. 98:1642-1649.

8. Sorbi, D., et al. 1996. Elevated levels of 92-kd type IV collagenase (matrix metalloproteinase 9) in giant cell arteritis. Arthritis Rheum. 39:1747-1753.
9. Rittner, H.L., et al. 1999. Tissue destructive macrophages in giant cell arteritis. Circ. Res. In press.

10. Poli, G., et al. 1985. Separation and characterization of the aldehydic products of lipid peroxidation stimulated by carbon tetrachloride or ADP-iron in isolated rat hepatocytes and rat liver microsomal suspensions. Biochem. J. 227:629-638.

11. Uchida, K., Szweda, L.I., Chae, H.Z., and Stadtman, E.R. 1993. Immunochemical detection of 4-hydroxynonenal protein adducts in oxidized hepatocytes. Proc. Natl. Acad. Sci. USA. 90:8742-8746.

12. Bohren, K.M., Bullock, B., Wermuth, B., and Gabbay, K.H. 1989. The aldoketo reductase superfamily. cDNAs and deduced amino acid sequences of human aldehyde and aldose reductases. J. Biol. Chem. 264:9547-9551.

13. Flynn, T.G., and Green, N.C. 1993. The aldo-keto reductases: an overview. Adv. Exp. Med. Biol. 328:251-257.

14. Gabbay, K.H., Merola, L.O., and Field, R.A. 1966. Sorbitol pathway: presence in nerve and cord with substrate accumulation in diabetes. Science. 151:209-210.

15. Bhatnagar, A., Liu, S.Q., Das, B., Ansari, N.H., and Srivastava, S.K. 1990. Inhibition kinetics of human kidney aldose and aldehyde reductases by aldose reductase inhibitors. Biochem. Pharmacol. 39:1115-1124.

16. Brack, A., et al. 1997. Glucocorticoid-mediated repression of cytokine gene transcription in human arteritis-SCID chimeras. J. Clin. Invest. 99:2842-2850.

17. Vinores, S.A., et al. 1988. Aldose reductase expression in human diabetic retina and retinal pigment epithelium. Diabetes. 37:1658-1664.

18. Brack, A., et al. 1997. Giant cell arteritis is a T cell dependent disease. Mol. Med. 3:530-543.

19. Grimshaw, C.E., and Mathur, E.J. 1989. Immunoquantitation of aldose reductase in human tissues. Anal. Biochem. 176:66-71.

20. Kern, T.S., and Engerman, R.L. 1982. Immunohistochemical distribution of aldose reductase. Histochem. J. 14:507-515.

21. Beyer-Mears, A., Diecke, F.P., Mistry, K., and Cruz, E. 1997. Comparison of the effects of Zopolrestat and Sorbinil on lens myo-inositol influx. Pharmacology. 54:76-83.

22. Esterbauer, H. 1993. Cytotoxicity and genotoxicity of lipid-oxidation products. Am. J. Clin. Nutr. 57:779S-785S.

23. Bohren, K.M., Grimshaw, C.E., and Gabbay, K.H. 1992. Catalytic effectiveness of human aldose reductase. Critical role of C-terminal domain. J. Biol. Chem. 267:20965-20970.

24. Robinson, B., Hunsaker, L.A., Stangebye, L.A., and Vander, J.D. 1993. Aldose and aldehyde reductases from human kidney cortex and medulla. Biochim. Biophys. Acta. 1203:260-266.

25. Ruepp, B., Bohren, K.M., and Gabbay, K.H. 1996. Characterization of the osmotic response element of the human aldose reductase gene promoter. Proc. Natl. Acad. Sci. USA. 93:8624-8629.

26. Wang, K., Bohren, K.M., and Gabbay, K.H. 1993. Characterization of the human aldose reductase gene promoter. J. Biol. Chem. 268:16052-16058.

27. Gabbay, K.H., and O'Sullivan, J.B. 1968. The sorbitol pathway. Enzyme localization and content in normal and diabetic nerve and cord. Diabetes. 17:239-243.

28. Gabbay, K.H., and Cathcart, E.S. 1974. Purification and immunologic identification of aldose reductases. Diabetes. 23:460-468.

29. Tomlinson, D.R. 1993. Aldose reductase inhibitors and the complications of diabetes mellitus. Diabet. Med. 10:214-230.

30. Pfeifer, M.A., Schumer, M.P., and Gelber, D.A. 1997. Aldose reductase inhibitors: the end of an era or the need for different trial designs? Diabetes. 46(Suppl. 2):S82-S89.

31. Srivastava, S., Chandra, A., Bhatnagar, A., Srivastava, S.K., and Ansari, N.H. 1995. Lipid peroxidation product, 4-hydroxynonenal and its conjugate with GSH are excellent substrates of bovine lens aldose reductase. Biochem. Biophys. Res. Commun. 217:741-746.

32. Vander, J.D., Torres, J.E., Hunsaker, L.A., Deck, L.M., and Royer, R.E. 1997. Physiological substrates of human aldose and aldehyde reductases. Adv. Exp. Med. Biol. 414:491-497.

33. Vander, J.D., et al. 1995. Substrate specificity of human aldose reductase: identification of 4-hydroxynonenal as an endogenous substrate. Biochim. Biophys. Acta. 1249:117-126.

34. Spycher, S., Tabataba-Vakili, S., O'Donnell, V.B., Palomba, L., and Azzi, A. 1996. 4-hydroxy-2,3-trans-nonenal induces transcription and expression of aldose reductase. Biochem. Biophys. Res. Commun. 226:512-516.

35. Nathan, C.F. 1982. Secretion of oxygen intermediates: role in effector functions of activated macrophages. Fed. Proc. 41:2206-2211.

36. Esterbauer, H., Schaur, R.J., and Zollner, H. 1991. Chemistry and biochemistry of 4-hydroxynonenal, malonaldehyde and related aldehydes. Free Radic. Biol. Med. 11:81-128.

37. Montgomery, J.A., Jette, M., Huot, S., and Des, R.C. 1993. Acyloin production from aldehydes in the perfused rat heart: the potential role of pyruvate dehydrogenase. Biochem. J. 294:727-733. 